\title{
Japanese Couples' Childbirth Experiences in Michigan: Implications for Care
}

\author{
SeonAe Yeo, RN, PhD, Michael Fetters, MD, MPH, MA, and Yukio Maeda, MA
}

\begin{abstract}
Background: Subtle cultural differences in the childbirth experience for expatriate Japanese couples living in southeast Michigan can sometimes cause conflicts between them and American health caregivers. The purpose of this study was to examine Japanese couples' perceptions and experiences of prenatal care and childbirth in a region of the United States, and to explore the implications for providing culturally competent care. Methods: In this qualitative study, in-depth interviews of 11 Japanese couples $(\mathrm{n}=$ 22) were conducted at an outpatient primary care clinic in southeast Michigan by a team of researchers fluent in the Japanese language and knowledgeable about the culture. Participants also completed a short questionnaire. Results: The major themes that emerged comprised perceptions related to a language barrier, ultrasonography, prenatal vitamin supplementation, episiotomy, epidural anesthesia, and practitioner-patient relationship. Throughout these six themes it was evident that Japanese health care professionals had difficulty accepting health care that was different from the care provided in their home country. Conclusion: The most striking finding of this study was the difficulty for health caregivers to provide culturally competent care. Although the program has strongly invested in health professionals and support staff who were fluent in Japanese and knowledgeable about Japanese culture, conflicts still occurred. (BIRTH 27:3 September 2000)
\end{abstract}

People who move from their place of birth to live in new countries or even new regions within their own country encounter many cultural differences in medical and nursing services and care (1-3). Investigation has shown that such differences may include recommended immunizations for children (4-8), prenatal care (9-11), and medical management for the same symptoms (12).

Clinically accessible overviews of cultural practices

\footnotetext{
SeonAe Yeo is Associate Professor of Nursing at the University of Michigan, School of Nursing; Michael Fetters is Assistant Professor of Family Medicine and Director of the Japanese Health Program at the University of Michigan, Medical School; and Yukio Maeda is a doctoral candidate at the University of Michigan, Department of Political Science, Ann Arbor, Michigan.
}

This investigation was made possible, in part, through the generous support of the University of Michigan Center for Japanese Studies, Ann Arbor, Michigan.

Address correspondence to SeonAe Yeo, PhD, RN, The University of Michigan, School of Nursing, 400 N. Ingalls, Room 3160, Ann Arbor, MI 48109-0482.

(C) 2000 Blackwell Science, Inc. and differences are helpful, but they may not contain sufficient detail to explain cultural differences that occur in community-based settings for events such as childbirth (13). The need for targeted research on specific cultural groups, and the opportunity for the authors to work closely with the expatriate Japanese population living in southeast Michigan, prompted our investigation.

The purpose of the study was to describe the role of cultural differences in childbirth for expatriate Japanese couples in a region of the United States, and to examine the implications for providing culturally competent care. Lavizzo-Mourey and Mackenzie have defined culturally competent care as health care that demonstrates awareness and integration of three population-specific issues, namely, health-related belief and cultural values, disease incidence and prevalence, and treatment efficacy (14).

\section{Methods}

The study was conducted in a university-affiliated outpatient clinic and its associated university hospital in 
southeast Michigan between November 1997 and July 1998. This clinic has an interdisciplinary team of staff fluent in Japanese, knowledgeable about the culture, and who operate a primary health care services program for Japanese-speaking people in the area.

About 5000 Japanese people (1\% of the total population) reside in the area where this study was conducted. Although the total number of Japanese people in the region has remained relatively constant, turnover is considerable, since many families are on temporary assignment. Whereas most make efforts to learn about United States culture and customs, inevitably they maintain many of the fundamental aspects of their Japanese lifestyle, such as their diet, their children's Japanese language education through a weekend school, and social circles. They are familiar with modern conveniences and are intensive users of hightechnology products. Most have been treated with "Western medicine" in Japan.

In this study ethnographic methods were employed to understand Japanese couples' experiences with the birthing process in the United States (15). The research protocol was approved by the Institutional Review Board of the University of Michigan, and all participants gave written informed consent in Japanese. The investigators reviewed their personal and professional backgrounds through the process of reflexivity (i.e., describing investigators' own experiences associated with childbirth) to identify their own biases (16-18).

Japanese couples, defined as having at least one of a couple who was Japanese born and spoke Japanese as a first language, who were clients in the outpatient clinic or attended Japanese birthing classes during the study period were eligible to participate. Participants were recruited during clinic visits or a prenatal class. Eleven pregnant Japanese couples $(n=22)$ participated in the study.

\section{Data Collection}

In-depth interviews were conducted, one prenatally and a second postnatally, within 4 weeks of delivery. The interview guide examined three domains: personal (intention and experience of pregnancy and birth, preparation/processing and perception of pregnancy); social (experience and perception about the life in the United States, tradition/ritual in birthing), and health care system (comparison of Japan and the United States). All members of the research team were bilingual, and the interviews were conducted in Japanese. Husbands and wives were interviewed separately. Additional questions were included in the course of the investigation to explore new issues raised by the participants' responses (16). Most prenatal interviews were conducted face-to-face, and most postpartum inter- views were conducted by means of the telephone. In addition to interviews, participants completed a 31item, self-administered questionnaire containing questions about demographics and childbearing history.

The investigators dictated observations from the monthly Japanese birthing classes, outpatient clinical encounters, and observations about the couples' experiences during the delivery. These were transcribed for use during the analysis.

\section{Data Analysis}

After multiple readings of the transcripts, the research team developed an initial series of themes based on the interviews and field notes. This process was employed to describe identifying analytical categories, utterance identifications, and observations; to expand and compare observations; and to develop themes and new empirically testable hypotheses. Based on this process, the major themes and subthemes were identified and responses that best illustrated these phenomena were selected for presentation. Demographic data were calculated for the survey questionnaires using the statistical package SPSS (19).

\section{Results}

\section{Sample Characteristics and Social Networks}

Twenty-two people participated in a total of 40 interviews. Nine couples participated in both prenatal and postpartum interviews, one couple participated only in a prenatal interview, and another couple participated only in a postpartum interview. The average age of the husbands was 35.2 years and that of the wives was 32.9 years. All participants graduated from high school, and the husbands and wives had an average of 5.7 years and 2.7 years of education after high school, respectively. The husbands in the study attained higher education levels than the average husband in Japan (20). Most participants (95.5\%) were from cities rather than rural areas. Three husbands and three wives were health care professionals in Japan, although none of them worked in this capacity in the United States.

Nineteen Japanese participants (86\%) came to the United States for the first time from Japan. The average length of the stay in the United States at the time of interview was 30 months ( $\mathrm{SD}=24)$, and participants planned to stay in the United States an average of 28 months $(\mathrm{SD}=23$ ) longer. Thus, the average length of the expected total stay in the United States in this cohort was 58 months (4.8 years). All participants lived with spouses and had been married an average of 5.2 years $(\mathrm{SD}=3)$.

Six couples had previously given birth to one or 
two children in Japan. Since 1970, the average number of children in each family in Japan has been 2.2 (20), although the birth rate has recently fallen to less than 1 infant per a woman of reproductive age. With respect to the participants' births in Japan, a husband had attended one delivery. When asked whether husbands planned to attend the upcoming births in the United States, all wives and all but one husband answered that the husband would attend the birth. The main reason for a husband's attendance, according to wives, was either that he had to interpret or the wife wanted him to accompany her. The main reason for a husband's attendance, according to the husbands, was that his attendance seemed to be expected in the United States.

Thirteen participants (59\%) indicated that someone (usually one of their mothers) would come from Japan to help them at the time of the birth, and four couples (41\%) could not arrange for a family member to help them out, even though couples were well aware of the importance of such support persons during the early postpartum period in a foreign country. Japanese women are usually reluctant to ask their Japanese friends who live nearby to help for an extended period.

\section{Major Themes}

Several major themes with medical and cultural implications emerged during interviews with participants.

\section{Language Barrier}

The language barrier affected all aspects of the care and medical treatment that these Japanese couples received, not only resulting in a lack of informed care and treatment but also misunderstanding and, in some cases, conflicts. Contrary to the general notion that language barriers exist only for those who are relatively less educated, our study indicated that even the husbands who had worked as physicians and researchers in Japan experienced formidable language barriers. One physician husband who was conducting research under an American scientist admitted:

\footnotetext{
English is still hard for me. If people speak slowly, I can understand to some extent. But I have a hard time to promptly answer what I want to say in English during conversation . . . . Even though I wrote medical charts in English when I was in Japan, it usually involved simple syntax only, without creating complex sentences.
}

Language barriers for the wives were much greater. Although the participants had already lived in the United States for an average of 2.5 years, many wives still lived in an exclusively Japanese society. They rarely socialized with American neighbors, and created a social network consisting only of other Japanese women in the area. Thus, most wives reported that their husbands were the primary interpreters whenever they visited clinics or hospitals. The husbands could understand their doctors' words most of the time except for medical technical terms, although the physician husbands usually had fewer problems with these terms than nonhealth professional participants. They also commented that often it was difficult to understand paramedical staff's comments.

All participants indicated that they could read English and often brought English-Japanese dictionaries with them. Since a common perception exists that it is easier for non-English-proficient patients to understand written materials than verbal discussion, it is an appealing idea to provide written material to minimize the difficulties of communication. However, in reality, they did not read the English handouts. Again, the comments from a physician husband during his prenatal interview negated this view:

\footnotetext{
I have not read any handouts or booklets handed to us at clinics. Both my wife and I read only magazines and books (related to childbirth preparation) sent to us from Japan. . . I I have been warned by my Japanese friends that I need to sign a lot of papers during labor and delivery. I never signed or was asked to read papers when I had our first son in Japan. It will be a different experience.
}

When treatment choices and care management were different from those experienced by these Japanese people in their culture, language barriers created more serious problems than simply frustration. When one couple's older son fell off a slide, the couple took him to the doctor, expecting to have him x-rayed, according to Japanese medical custom. One American doctor examined the child but did not do an x-ray examination. Later, the couple found out that an x-ray film ordered by a second doctor revealed a broken bone. The father was frustrated with this "mistreatment," and was angry with himself because he could not convince the first doctor to do an x-ray examination due to the language barrier.

\section{Ultrasonography}

The Japanese medical community provides parents with fetal sonograms at each prenatal visit. It has become a ritual for parents to start with a series of fetal ultrasound photos in their baby's first photo album. Japanese physicians and couples believe that fetal ultrasound examinations permit surveillance for normal fetal development. Since this practice is the norm in Japan, even couples who never had children in Japan had the same expectation of receiving regular ultrasound examinations in the United States. Many participants, including physicians and nurses, expressed 
anxiety about not having a sonogram at routine prenatal visits. Those who had previously given birth in Japan were especially dumbfounded. Parenthetically, normal births are not covered by health insurance (national or private) in Japan. Parents bear most of the cost for prenatal care, including every ultrasound examination, as well as the costs incurred during labor and birth. Government programs cover some of prenatal care visits and laboratory fees.

One mother who moved to the United States during the sixth month of her pregnancy was particularly anxious about not having regular ultrasound examinations of her baby. While in Japan, she felt assured of the normal development of the fetus by looking at a sonogram at each prenatal visit. Moving to a totally unfamiliar location where she did not understand the language provoked anxiety about her baby's health. Together with many other uncertainties of living in a foreign country, the inability to see her fetus's picture in the American doctor's office added to her general anxiety.

I had a lot of anxiety during prenatal visits because there was no ultrasound . . . I should feel OK since I heard my baby's heart sound, but I still wanted to know if the placenta was located at the right place.

The interview with her husband further revealed her emotional difficulty:

\footnotetext{
(During the pregnancy), it was really hard, you know, I needed to work . . . and when I came home, often I found my wife under the weather, depressed. Doubt came to my mind repeatedly about whether we could really do this (without any help). I was worried. She cried quite often. . . . Yes, she cried often because she worried about the baby and perhaps many other things . . . you know, still I had to go to work. I could not stay with her at home . . . I had to go to work.
}

Her need for seeing the sonograms of her baby, whether to fulfill cultural or emotional needs, was never discussed with her doctor.

\section{Prenatal Vitamin Supplementation}

Whereas a daily prenatal vitamin with folic acid is routinely prescribed in the United States, this is not the case in Japan. Iron supplements are prescribed only if iron deficiency anemia is identified. Many participants reported taking a prenatal vitamin as one of the most puzzling and uncomfortable instructions during the pregnancy, and Japanese couples regularly resisted doctors' orders to comply. In their mind, taking a vitamin supplement rather than eating a nutritionally balanced meal was believed to be a shortcut and incompatible with ideal motherhood. Japanese people take great pride in their eating habits. There is a gener- ally accepted notion that eating sufficient nutrients through healthy homemade meals best promotes normal fetal growth and development. Japanese participants believed that eating "real" food and certain "good" foods (e.g., seaweed or small dried whole fish called chirimenjako) showed that one was a good mother. Some Japanese couples seemed to be almost offended by being encouraged to take a prenatal vitamin. For example, one mother who was a health care practitioner in Japan questioned the necessity of the vitamin supplement prescribed by her prenatal caregiver:

I have been eating Japanese food in the United States just like I did in Japan when I had my first child. I never took a vitamin with my first child . . . and it did not have any bad effects on my child ... then American doctors told me that it's better to take vitamins . . . I don't mind taking it, but I don't know why I need to take it, as nothing bad happened with my first child in Japan.

Her caregiver explained that large-scale epidemiologic studies, including studies from Oriental patients in China (20-23), showed that folic acid prevents neural tube defects and anencephaly. In response to this explanation, she asked:

\begin{abstract}
Well, then, is there a greater incidence of neural tube defects in Japan? . . . I was working at a pediatric ward in Japan. I rarely saw neural tube defects . . . I don't mind taking it, but I just don't think I need it. I wonder whether Americans eat balanced meals. I don't know if they eat enough vegetables. I believe that it is better to take necessary nutrients through meals rather than taking vitamin supplements.
\end{abstract}

Despite repeated attempts to understand her resistance to taking a prenatal vitamin, it was only later during her in-depth interview that the source of her resistance was identified.

Another important cultural issue relating to vitamin supplementation is that Japanese people perceive prenatal vitamins to be medicine (since they are prescribed by physicians), and a strong belief exists that pregnant women should avoid taking medications at all costs. One mother told us during her postpartum interview:

I could not understand why I had to take this stuff. A booklet said that vitamins are not medication. But to me, they are medicine because they are tablets in a medicine bottle . . . I was not sure what my doctor was treating with this medicine ... If I were in Japan, doctors would have said to eat balanced meals instead of prescribing medication.

Some mothers declared that they hated taking medication during pregnancy. Although such strong resistance existed against taking vitamins prenatally, they were willing to take vitamins postnatally, but only because their Japanese friends, rather than a physician or nurse, said that this supplementation helped increase 
the volume of breastmilk. One woman reported that her obstetrician in Japan instructed her to stop taking the prenatal vitamin.

\section{Episiotomy}

A participant at 30 weeks' gestation found out that her physician did not recommend or routinely perform an episiotomy. In her training as a health care practitioner in Japan, the performance of an episiotomy was a scientific procedure taught and practiced by obstetricians. She was dumbfounded and panicked because the medical practices were in distinct conflict with the training she had received in Japan. She believed that an episiotomy would prevent tears and that a straight incision would heal much faster than a tear. As was the case with the folic acid supplementation, the physician hoped the scientific background of the woman and her husband in the health care professions would respond to epidemiologic evidence demonstrating worse outcomes when episiotomy was used $(12,24,25)$. Although episiotomy was never considered as an issue among nonhealth-care worker participants, the doctor's resistance to performing an episiotomy compromised this Japanese woman's trust in him. She abruptly switched to another doctor who promised to cut her perineum.

In Japan, primiparas receive episiotomy. Multiparas, if the perineum extends well, may not receive episiotomy. I don't mind pain, but it is much better to be cut than be torn.

Her medical cultural beliefs from Japan were so strong and ingrained that she transferred her care. Despite the reluctant agreement by her original doctor to provide an episiotomy, his lack of enthusiasm for episiotomy so profoundly conflicted with her belief that routine episiotomy was the best care that she did not feel comfortable continuing care with him.

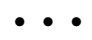

Despite the preceding tribulations, participants perceived a series of cultural differences positively.

\section{Epidural Analgesia}

Epidural anesthesia is not routinely offered in the practice of obstetrics in Japan. Women have been acculturated to accept childbirth-related pain. Although Japanese women accept the pain, they are not free from fear associated with pain. Many women (both primiparas and multiparas) discussed the psychological comfort of having a backup, just in case the pain might become intolerable. On the other hand, women seemed to have concerns about side effects of the analgesia on the baby. When they were asked to talk about epidural analgesia, many participants expressed both emotions. Although they wanted to "experiment" with a new method that might dramatically reduce their pain, expressing a desire for no pain seemed to be somewhat difficult. Interestingly, a few second-time mothers said that they wanted to try, since this would be the second child.

Remarks from postpartum interviews gave further insights. One second-time mother said that when her pain became intolerable, she requested an epidural. She could not believe how peaceful and happy she was during the labor, compared with her first son's birth. She discovered that epidural analgesia did not completely eliminate her labor pain. She was thrilled that she could feel some pain, as if she was afraid to lose all pain. She thought that, because of the epidural analgesia, she reserved energy to share the joy of greeting her new daughter.

Some women admitted that they wanted to try this "new method," but their husbands asked them not to use it because of their own concerns for fetal wellbeing. One husband in this group was a physician. No wife expressed anger over her husband's request. These women perceived their husbands' decision to be proof of being a caring father, which is often synonymous with being a good husband.

\section{Caregiver-Client Relationship}

These Japanese couples thought that United States doctors and nurses were highly professional, and often contrasted their experiences with American and Japanese doctors. Many, including some who were physicians in Japan, perceived that Japanese doctors were authoritative and overbearing, taking their hierarchical superiority for granted and acting almost oppressively when dealing with patients. American doctors were perceived as more democratic, open, and jovial. For example, the husband of a couple who underwent infertility treatment in the United States and Japan said that the treatment for infertility was stigmatized in Japan.

\footnotetext{
(Treatment of infertility involved) ... not only the level of medical science, but the social environment. My company gave me very little freedom to arrange visits to a clinic in Japan. My work here was flexible enough to arrange visits without feeling guilty. We were much more relaxed here. We would not have had a child, had we stayed in Japan.
}

Among participants it was almost unanimous that their encounters with American doctors made Japanese couples realize how uncaring many Japanese doctors were. That, at least, was the couples' perceptions, even though they often did not understand many things their American doctors told them. 
Nurses were supportive and friendly, yet knowledgeable, and provided humane and timely care, according to participants. Japanese couples also expressed admiration for highly efficient American teamwork. One woman who worked as a health care practitioner in Japan admitted that the quality of the care she received here would never be available in Japan.

Nurses were attentive to me all the time I spent in the labor and delivery room. That never happens in Japan. They (American nurses) were very professional. In Japan, staff shortages were permanent. The phrase most frequently used by nurses in Japan is, "Wait for a second, I'll be there soon." I often attended more than one birth simultaneously. That scared me as I didn't know what to do if something bad happened to multiple patients at the same time.

Overall, participants described Japanese nurses as relatively lacking in the capacity to recognize individual needs or provide individualized care compared with American nurses. In contrast, the perception was that Japanese nurses work like factory-line workers. Whether or not these differences were the result of different health care delivery systems in the two countries or different cultural values held by the two societies was not discussed during the interviews.

\section{Discussion}

The most striking finding of this research was the difficulty for caregivers to provide culturally competent care. Most study participants were patients in a program dedicated to the care of Japanese-speaking people. Although this Michigan program had strongly invested in health professionals and support staff who were fluent in Japanese and knowledgeable about the culture, conflicts still occurred. This study revealed that health-related beliefs about treatment efficacy cultivated in modern health care systems can impose as strong an impact as those held by traditional healers.

First, language barriers created problems. Although the program includes receptionists, nurses, a social worker, and physicians, patients sometimes must still use English to interact with the system and their environment. Since the program's inception, efforts to obtain educational materials in Japanese have been ongoing to reduce these barriers.

Second, health insurance reimbursement policies influence the content of clinical practice in the United States. For example, the relatively low cost in Japan and the high cost in the United States of ultrasonography, or the much shorter duration of hospital stay in the United States than in Japan, cannot be overcome simply by enhanced understanding of the culture by the caregiver. Rather, it may be necessary to identify the key person who is recognized as a medical authority in the community and to educate patients about these differences through the key person.

Third, medical practices are strongly divergent, and beliefs about scientifically valid practices are strongly instilled. The effect is surprisingly stronger with Japanese people who are themselves health care professionals in their homeland. To our surprise, Japanese health care practitioners experienced much more difficulty accepting American health care than Japanese who held nonhealth-care occupations. Because they were health care practitioners trained in modern "Western" medicine, everyone assumed that a shared scientific framework would exist about the practice of Western medicine. It is naïe for health care practitioners to believe that medical science is universal. That which is believed to be science often is merely established medical routine without empirical or scientific evidence to support it. Our experience in trying to work with these Japanese health professionals by providing evidence was, at best, no help in convincing them about an evidence-based approach and, at worst, may have exacerbated the situation.

Fourth, ethical dilemmas can arise in endeavors to provide culturally competent care. In the Japanese program where the authors work, the philosophy is to conform with any reasonable practice thought to be helpful, or at least not harmful. Patient demand for a practice such as routine episiotomy, which is commonly used in Japan, raised an ethical dilemma for the caregiver, given his or her strongly held belief to do no harm. Similarly, the imperative to encourage prenatal vitamins with folic acid to benefit the pregnant woman by preventing neural tube defects in the fetus is clear. Creative mechanisms, such as identifying the medical authority in the community, to encourage women to take prenatal vitamins may be necessary. For example, in one prenatal class we demonstrated how much vegetable a woman would have to eat on a daily basis to acquire the recommended amount of folate. The volume and variety of foods needed seemed helpful for convincing some women to take the prenatal vitamins, since they often found out that the daily amount of ingested food contained only 2 to $6 \mathrm{mg}$ of iron.

\section{Implications for Care}

We propose some suggestions that will enable practitioners to provide more culturally sensitive care with ultimately lower health care costs. First, it is clear that no caregiver should assume that Japanese childbearing couples will read and understand written materials, no matter how well they seem to understand English. If 
handouts are not available in Japanese, one should choose the most summarized, shortened, clearly written (without idioms) materials, using as many graphs and pictures as possible. Interpreters should be available whenever possible.

Second, caregivers must keep in mind that although routine medical protocols might have been developed based on guidelines from authorities or scientific evidence, implementing the protocols may require the accommodation of culturally different health care practices. We believe that such a policy is the preferred approach if it will not cause harm through an act of commission or omission. Depending on the situation, the caregiver should try either to adjust the protocol to meet the client's health care expectations or to explain the rationale for the protocol to the client. In the latter case, the caregiver should not underestimate an active effort to elicit the woman's concerns and fears.

Third, regrettably, existing health insurance systems in the United States usually will not understand or pay for culturally specific practices requested by the client, especially those such as ultrasound that may be reasonable if patient-centered outcomes are considered. Couples can be given the option of paying out of pocket for services not routinely covered by health policies. In our experience, most women have been reluctant to pay for ultrasound examinations, although some have paid out of pocket.

Fourth, Japanese women are accustomed to following dietary guidelines rather than taking vitamin supplements, and they are often willing to learn nutritional information about unfamiliar foods (beets, cereal, different kinds of cheese, etc.). Most Japanese expatriates keep their food customs, even if the ingredients have to be shipped from Japan. This tendency is intensified during pregnancy. In the Japanese family health program prenatal class, led by the principal author, many American foods have been incorporated, providing nutritional data together with the data for Japanese food in both English and Japanese. This procedure seemed to mediate tension between physicians and Japanese clients with respect to the vitamin supplement dispute.

This study adds to the body of medical and social science literature demonstrating the pervasive nature of language barriers and deeply held cultural beliefs $(2,14,26)$. Interestingly, many reports of cultural conflicts are about members of cultural groups who have not been exposed to Western medicine and who possess no command of the majority language. Japan can boast of several impressive measures of health. The infant morbidity and mortality rates are the lowest in the world, and average longevity is extremely high. It is eye-opening to discover that people, especially health professionals, from one of the most industrialized na- tions experience so much difficulty when receiving health care in another industrialized nation. Even in a program designed specifically to accommodate cultural needs, it will take greater efforts than those already established to provide culturally sensitive care.

\section{Acknowledgment}

The authors wish to thank all the families, staff, nurses, and doctors who contributed to this research. We are indebted to the Japanese couples who participated in this study for their patience with our endless inquiries and follow-up.

\section{References}

1. Kleinman A. Ethnicity and clinical care: The Chinese patient. Physician Assist Health Pract 1980;4(1):60-68.

2. Fadiman A. The Spirit Catches You and You Fall Down: A Hmong Child, Her American Doctors, and the Collision of Two Cultures. New York: Farrar, Straus, and Giroux, 1997.

3. Freude G, Ullsperger P, Molle M. Assessment of mental effort by means of slow brain potentials. Electroencephalogr Clin Neurophysiol Suppl 1995;44:297-301.

4. Ada G. Global aspects of vaccination. Int Arch Allergy Immunol 1995;108(4):304-308.

5. Grob PJ. Report on Working Group 2: Austria, Belgium, Bulgaria, Germany, Greece, Hungary, Malta, Russia, Switzerland, Turkey and Uzbekistan [published erratum in Vaccine 1999;17(19):2472]. Vaccine 1998;16(suppl):S61-S62.

6. Bonanni P. Report on Working Group 1: Albania, Andorra, Canada, France, Italy, Moldova, Portugal, Poland, Romania and Spain. Vaccine 1998;16(suppl):S58-S60.

7. Kane MA. Status of hepatitis B immunization programs in 1998. Vaccine 1998;16 (suppl):S104-S108.

8. Japanese encephalitis vaccines. Wkly Epidemiol Rec 1998; 73(44):337-344.

9. Samson L, King S. Evidence-based guidelines for universal counseling and offering of HIV testing in pregnancy in Canada [published erratum in CMAJ 1998;159(1):22]. CMAJ 1998; 158(11):1449-1457.

10. Weissman AM. Preventive health care and screening of Latin American immigrants in the United States. J Am Board Fam Pract 1994;7(4):310-323.

11. Okafor CB. Availability and use of services for maternal and child health care in rural Nigeria. Int J Gynaecol Obstet 1991; 34(4):331-346.

12. Maduma-Butshe A, Dyall A, Garner P. Routine episiotomy in developing countries: Time to change a harmful practice. BMJ 1998;316:1179-1180.

13. Lynch EW, Hanson MJ. The importance of cross-cultural effectiveness. Caring 1992;11(10):14-19.

14. Lavizzo-Mourey R, Mackenzie ER. Cultural competence: Essential measurements of quality for managed care organizations. Ann Intern Med 1996;124(10):919-921.

15. Bernard HR. Field notes: How to take, code, and manage them. In: Bernard HR, ed. Research Methods in Anthropology: Qualitative and Quantitative Approaches. 2nd ed. Walnut Creek, CA: AltaMira Press, 1995:180-207

16. Ventres WB, Frankel RM. Ethnography: A stepwise approach for primary care researchers. Fam Med 1996;28(1):52-56. 
17. Crabtree BF, Miller WL. A qualitative approach to primary care research: The long interview. Fam Med 1991;23(2): $145-151$.

18. Olesen V. Feminisms and models of qualitative research. In: Denzin N, Lincoln Y, eds. Handbook of Qualitative Research. Thousand Oaks, CA: Sage, 1994:158-174.

19. Statistical Package for Social Science. SPSS 7.5 for Windows. Chicago: Author, 1995.

20. National Institute of Population Study. The 11th Census Report: National Survey on Marriage and Birth; Summary Report of Marriage Survey. Tokyo: Author, 1997.

21. Berry RJ, Li Z, Erickson JD, et al. Prevention of neural-tube defects with folic acid in China. China-U.S. Collaborative Project for Neural Tube Defect Prevention. $N$ Engl J Med 1999;341;1485-1490.
22. Czeizel AE, Dudas I. Prevention of the first occurrence of neural-tube defects by periconceptional vitamin supplementation. N Engl J Med 1992;327:1832-1835.

23. MRC Vitamin Study Research Group. Prevention of neural tube defects: Results of the Medical Research Council Vitamin Study. Lancet 1991;338:131-137.

24. Lede RL, Belizan JM, Carroli G. Is routine use of episiotomy justified? Am J Obstet Gynecol 1996;174:1399-1402.

25. Argentine Episiotomy Trial Collaborative Group. Routine vs selective episiotomy: A randomised controlled trial. Lancet 1993;342:1517-1518.

26. Kleinman A, Kunstadter P, Alexander ER, Gale JL, eds. Culture and Health in Asian Societies: Anthropological, Psychiatric and Public Health Studies. Cambridge, MA: Schenkman, 1978. 